\title{
The screening for chronic kidney disease among older people across Europe (SCOPE) project: findings from cross-sectional analysis
}

\author{
Andrea Corsonello $^{1^{*}}$, Ellen Freiberger ${ }^{2}$ and Fabrizia Lattanzio ${ }^{1}$
}

\section{Editorial}

The scenario of CKD in the older population is known to be very complex. Among community dwelling older people there is a broad range of different functional levels ranging from fitness and robustness to frailty or disability. Impaired physical function, frailty and disability, cognitive impairment, depression, vision and hearing impairment, malnutrition, and sarcopenia all contribute to worsen health outcomes and to increase the use of health care resources, thus challenging health care systems. The benefits of preventing/slowing the progression of CKD among these patients have the potential to impact different social and health domains, e.g. reducing the need for long-term care and the cost related to caregiving. Thus, early identification of CKD represents a relevant issue to be addressed in the older population.

At the end of 2013 the American College of Physicians (ACP) issued a statement against the usefulness of chronic kidney disease (CKD) screening for the general adult population [1]. Those recommendations were severely criticized by nephrologists, fully aware that early detection and intervention can slow the progression of CKD to renal failure. Noting the lack of involvement of nephrologists in the development of ACP statements, Molitoris claimed that "We should be committed to supporting all approaches that will change kidney disease from a silent disease too often discovered in its late stages to one that is identified when modifications can affect the progression of the disease [ ... ]. The entire medical

\footnotetext{
* Correspondence: a.corsonello@inrca.it

${ }^{1}$ Italian National Research Center on Aging (IRCCS INRCA), Ancona, Fermo and Cosenza, Cosenza, Italy

Full list of author information is available at the end of the article
}

community must act sooner and enlist patients' help in minimizing the comorbidities and progression that too often remain undetected until very late in the disease process" [2].

CKD impact on global health, either as a risk factor for morbidity and mortality or by causing cardiovascular disease. A recent systematic analysis of the Global Burden of Disease Study 1990-2017 [3] showed that over the past 27 years CKD burden has not declined to the same extent as many other important non-communicable diseases. However, CKD and its consequences remain largely preventable and treatable and deserves greater attention in global health policy decision making. Indeed, several studies suggest that screening for CKD in high-risk and older populations may represent a cost-effective approach to reduce progression to renal failure and CKD mortality [4-7].

Current screening measures rely on creatinine-based estimated glomerular filtration rate (eGFR). They are known to have some degree of inaccuracy when used in older people and to carry an increased risk of both overand under-diagnosis, but alternative filtration markers have been identified and are worth of testing. Indeed, a screening program should take into account the characteristics of the target population to achieve effective results. A high proportion of older CKD patients are usually affected by multimorbidity, polypharmacy, frailty, functional and cognitive impairment and disability [816]. However, CKD screening programs to date have not included the use of the comprehensive geriatric assessment (CGA), the only assessment technology able to capture the numerous dimensions of health status and their complex interactions in older people. Accordingly, comparing different CKD screening methods for older 
complex patients in regards to their accuracy is considered a very important issue to be addressed. Indeed, this view is widely shared by the geriatric and nephrologist communities $[17,18]$, and the implementation of innovative findings is expected to improve CKD screening and management in such a vulnerable population (Fig. 1).

All these aspects have been the primary motivator for the development of a EU funded research project, titled "Screening for CKD among Older People across Europe (SCOPE)", aiming at comparing different screening methods for CKD in the older population, including a comprehensive set of CGA variables to explore population-specific outcomes [19].

Initial results from the baseline database of the SCOPE project [19] have been made available for the participating researchers in this special issue as a first description of the population studied, a cohort of 2464 patients from 7 countries, through a series of cross-sectional analyses about typically geriatric topics.

The main finding from the study by Moreno-Gonzalez et al. is the relevant prevalence of sarcopenia observed among community-dwelling people aged 75 years and older, using the most recent diagnostic criteria for sarcopenia endorsed by the EWGSOP2 consensus. Participants within poorer eGFR categories, irrespective of the equation used for its calculation, and albuminuria have a higher prevalence of sarcopenia and are more often severely sarcopenic. However, there are some differences in prevalence according to the eGFR formula used.

In the paper by Guligowska et al., the SCOPE study population shows generally features of overweight and obesity with small prevalence of malnutrition. Higher weight, circumferences, body mass index (BMI) and waist-to-height ratio (WHtR) were significantly associated with increased likelihood of having CKD, whilst higher Mini Nutritional Assessment (MNA) score and higher serum albumin were inversely associated with CKD. The risk of MNA $<24$ or hypoalbuminemia was increased among patients with estimated glomerular filtration rate $(\mathrm{eGFR})<30$ as compared to those with eGFR $>60 \mathrm{ml} / \mathrm{min} / 1.73 \mathrm{~m}^{2}$.

Corsonello et al. showed that CKD contributes significantly to multimorbidity patterns in a population of older outpatients and it was rarely observed without any co-occuring disease. The most significant co-occuring pairs involving CKD included hypertension, anemia, CHF, atrial fibrillation, myocardial infarction, hip fracture, and to a lesser extent hearing impairment, diabetes and cancer. Cluster analysis showed that CKD may cluster with hypertension and sensory impairments. CKD severity and physical performance as assessed by the Short Physical Performance Battery (SPPB) test may be associated with not negligible changes in both co-occuring pairs and multimorbidity clusters. These findings strengthen the need of including disease severity in multimorbidity studies. Additionally, assessing physical function and investigating interventions targeting physical function among complex multimorbid patients represents a research priority to improve outcomes and reduce costs associated with multimorbidity.

Britting et al. showed that CKD, at least in the early stages, is not a predictor of falls and injurious falls, whereas urinary incontinence measured by lower urinary tract symptoms (LUTS) increases the risk of falls and injurious falls. Nevertheless, the development of prospective analysis within the SCOPE study will help to clarify the association between CKD and falls. Additionally, these findings suggest that treating LUTS and physical limitation in older CKD patients might be an appropriate way to reduce risk of falling.

In the paper by Tap et al. cognitive impairment and depressive symptoms were not more prevalent among community-dwelling older persons in more advanced stages of CKD than in those without or in earlier stages of CKD. Kidney function was comparable in those with and without any signs of cognitive or mood disorders. The identification of CKD as modifiable risk factor for cognitive impairment and depressive symptoms in late

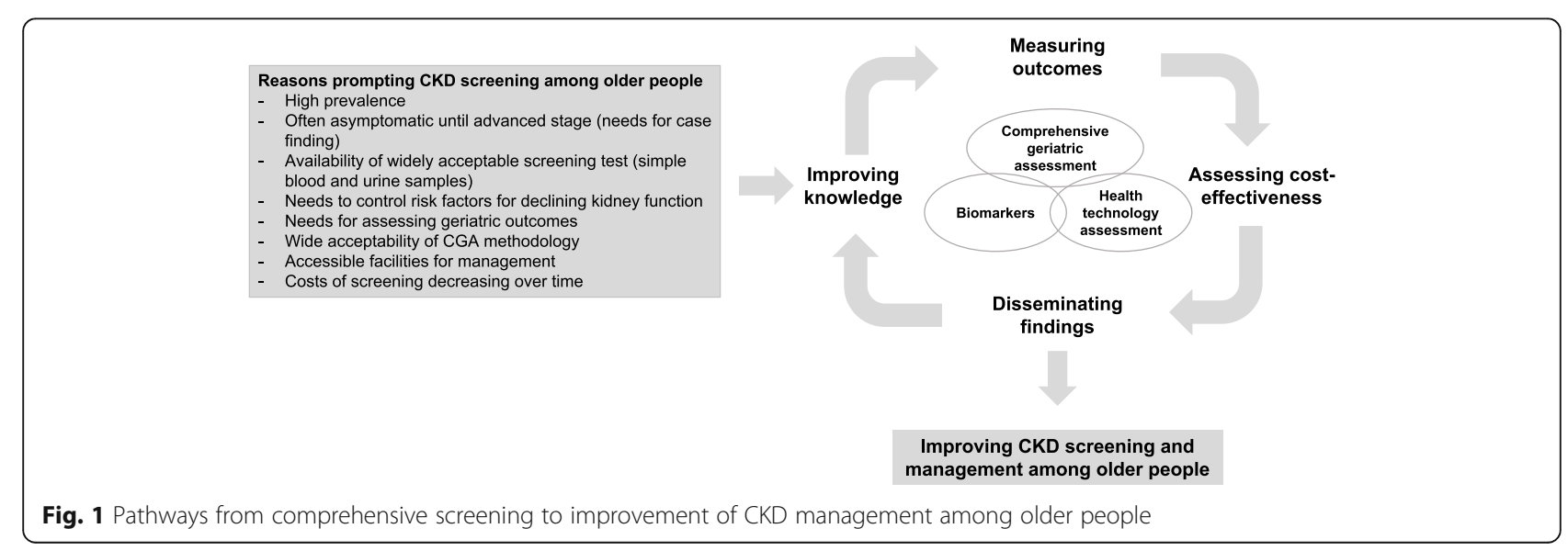


life might be relevant in order to optimize therapeutic strategies. Even in this case, longitudinal studies might give additional information on the possible effect of kidney function and/or CKD progression on mental health in late life.

Artzi-Medvedik et al. showed that CKD and its severity may be significantly associated with impaired Quality of Life (QoL) among community-dwelling older people, even after adjusting for several confounders. Additionally, physical performance and comorbidities were found to affect the association between CKD and QoL, which suggest that efforts should be made to decrease the effects of such modifiable risk factors in an attempt to improve QoL of CKD patients.

Taken together, the above studies prompt the assessment of nutritional status, sarcopenia, falls, mental health, quality of life, multimorbidity and physical function, and may be warranted in the usual care of older people with impaired kidney function, which could allow for their early detection and trigger proper interventions.

\section{Acknowledgements}

SCOPE study investigators.

Coordinating center, Fabrizia Lattanzio, Italian National Research Center on Aging (IRCCS INRCA), Ancona, Italy - Principal Investigator. Andrea Corsonello, Silvia Bustacchini, Silvia Bolognini, Paola D'Ascoli, Raffaella Moresi, Giuseppina Di Stefano, Cinzia Giammarchi, Anna Rita Bonfigli, Roberta Galeazzi, Federica Lenci, Stefano Della Bella, Enrico Bordoni, Mauro Provinciali, Robertina Giacconi, Cinzia Giuli, Demetrio Postacchini, Sabrina Garasto, Annalisa Cozza, Francesco Guarasci, Sonia D'Alia - Italian National Research Center on Aging (IRCCS INRCA), Ancona, Fermo and Cosenza, Italy - Coordinating staff. Romano Firmani, Moreno Nacciariti, Mirko Di Rosa, Paolo Fabbietti - Technical and statistical support. Participating centers - Department of Internal Medicine, Medical University of Graz, Austria: Gerhard Hubert Wirnsberger, Regina Elisabeth Roller-Wirnsberger, Carolin Herzog, Sonja Lindner

- Section of Geriatric Medicine, Department of Internal Medicine, Erasmus MC, University Medical Center Rotterdam, The Netherlands: Francesco Mattace-Raso, Lisanne Tap, Gijsbertus Ziere, Jeannette Goudzwaard.

- Department of Geriatrics, Healthy Ageing Research Centre, Medical University of Lodz, Poland: Tomasz Kostka, Agnieszka Guligowska, Łukasz Kroc, Bartłomiej K Sołtysik, Małgorzata Pigłowska, Agnieszka Wójcik, Zuzanna Chrząstek, Natalia Sosowska, Anna Telążka, Joanna Kostka, Elizaveta Fife, Katarzyna Smyj, Kinga Zel.

- The Recanati School for Community Health Professions at the faculty of Health Sciences at Ben-Gurion University of the Negev, Israel: Rada ArtziMedvedik, Yehudit Melzer, Mark Clarfield, Itshak Melzer; and Maccabi Healthcare services southern region, Israel: Rada Artzi-Medvedik, Ilan Yehoshua, Yehudit Melzer.

- Geriatric Unit, Internal Medicine Department and Nephrology Department, Hospital Universitari de Bellvitge, Institut d'Investigació Biomèdica de Bellvitge - IDIBELL, L'Hospitalet de Llobregat, Barcelona, Spain: Francesc Formiga, Rafael Moreno-González, Xavier Corbella, Yurema Martínez, Carolina Polo, Josep Maria Cruzado.

- Department of Geriatric Medicine, Hospital Clínico San Carlos, Madrid: Pedro Gil Gregorio, Sara Laínez Martínez, Mónica González Alonso, Jose A. Herrero Calvo, Fernando Tornero Molina, Lara Guardado Fuentes, Pamela Carrillo García, María Mombiedro Pérez.

- Department of General Internal Medicine and Geriatrics, Krankenhaus Barmherzige Brüder Regensburg and Institute for Biomedicine of Aging, Friedrich-Alexander-Universität Erlangen-Nürnberg, Germany: Alexandra Renz, Susanne Muck, Stephan Theobaldy, Andreas Bekmann, Revekka Kaltsa, Sabine Britting, Robert Kob, Christian Weingart, Ellen Freiberger, Cornel Sieber.
- Department of Medical Sciences, Uppsala University, Sweden: Johan Ärnlöv, Axel Carlsson, Tobias Feldreich.

We thank the BioGer IRCCS INRCA Biobank for the collection of the SCOPE samples.

Scientific advisory board (SAB).

Roberto Bernabei, Catholic University of Sacred Heart, Rome, Italy.

Christophe Bula, University of Lausanne, Switzerland.

Hermann Haller, Hannover Medical School, Hannover, Germany.

Carmine Zoccali, CNR-IBIM Clinical Epidemiology and Pathophysiology of Renal Diseases and Hypertension, Reggio Calabria, Italy.

Data and Ethics Management Board (DEMB).

Dr. Kitty Jager, University of Amsterdam, The Netherlands.

Dr. Wim Van Biesen, University Hospital of Ghent, Belgium.

Paul E. Stevens, East Kent Hospitals University NHS Foundation Trust,

Canterbury, United Kingdom.

\section{About this supplement}

This article has been published as part of BMC Geriatrics Volume 20 Supplement 1 2020: The Screening for Chronic Kidney Disease among Older People across Europe (SCOPE) project: findings from cross-sectional analysis. The full contents of the supplement are available at https://bmcgeriatr.biomedcentral.com/articles/supplements/volume-20-supplement-1

\section{Authors' contributions}

AC, EF and FL coordinated the Special Issue, conceived the Editorial and participated in manuscript drafting and revising. All authors read and approved the final manuscript

\section{Funding}

SCOPE study and publication costs are funded by the European Union Horizon 2020 program, under the Grant Agreement n 634869. Funding body had no role in the design of the study and collection, analysis, and interpretation of data, writing the manuscript and in the decision to publish the results.

Availability of data and materials

Not applicable.

Ethics approval and consent to participate

Not applicable.

\section{Consent for publication}

Not applicable.

\section{Competing interests}

The authors declare that they have no competing interests.

\section{Author details}

${ }^{1}$ Italian National Research Center on Aging (IRCCS INRCA), Ancona, Fermo and Cosenza, Cosenza, Italy. ${ }^{2}$ Department of Internal Medicine-Geriatrics, Institute for Biomedicine of Aging (IBA), Friedrich-Alexander-Universität Erlangen-Nürnberg, Erlangen, Germany.

Published: 2 October 2020

\section{References}

1. Qaseem A, Hopkins RH Jr, Sweet DE, Starkey M, Shekelle P. Clinical guidelines Committee of the American College of P: screening, monitoring, and treatment of stage 1 to 3 chronic kidney disease: a clinical practice guideline from the American College of Physicians. Ann Intern Med. 2013; 159(12):835-47.

2. Molitoris BA. Screening: screening for kidney disease--a lost opportunity. Nat Rev Nephrol. 2014;10(1):6-8.

3. Collaboration GBDCKD. Global, regional, and national burden of chronic kidney disease, 1990-2017: a systematic analysis for the global burden of disease study 2017. Lancet. 2020;395(10225):709-33.

4. Komenda P, Ferguson TW, Macdonald K, Rigatto C, Koolage C, Sood MM, Tangri N. Cost-effectiveness of primary screening for CKD: a systematic review. Am J Kidney Dis. 2014;63(5):789-97. 
5. Go DS, Kim SH, Park J, Ryu DR, Lee HJ, Jo MW. Cost-utility analysis of the National Health Screening Program for chronic kidney disease in Korea. Nephrology (Carlton). 2019;24(1):56-64.

6. Howard K, White S, Salkeld G, McDonald S, Craig JC, Chadban S, Cass A. Cost-effectiveness of screening and optimal management for diabetes, hypertension, and chronic kidney disease: a modeled analysis. Value Health. 2010;13(2):196-208.

7. Kondo M, Yamagata K, Hoshi SL, Saito C, Asahi K, Moriyama T, Tsuruya K, Yoshida H, Iseki K, Watanabe T. Cost-effectiveness of chronic kidney disease mass screening test in Japan. Clin Exp Nephrol. 2012;16(2):279-91.

8. Lattanzio F, Corsonello A, Abbatecola AM, Volpato S, Pedone C, Pranno L, Laino I, Garasto S, Corica F, Passarino G, et al. Relationship between renal function and physical performance in elderly hospitalized patients. Rejuvenation Res. 2012;15(6):545-52.

9. Roshanravan B, Khatri M, Robinson-Cohen C, Levin G, Patel KV, de Boer $\mathrm{H}$, Seliger S, Ruzinski J, Himmelfarb J, Kestenbaum B. A prospective study of frailty in nephrology-referred patients with CKD. Am J Kidney Dis. 2012; 60(6):912-21.

10. Fried LF, Lee JS, Shlipak M, Chertow GM, Green C, Ding J, Harris T, Newman AB. Chronic kidney disease and functional limitation in older people: health, aging and body composition study. J Am Geriatr Soc. 2006;54(5):750-6.

11. Pedone C, Corsonello A, Bandinelli S, Pizzarelli F, Ferrucci L, Incalzi RA. Relationship between renal function and functional decline: role of the estimating equation. J Am Med Dir Assoc. 2012;13(1):84 e11-84.

12. Kurella M, Chertow GM, Fried LF, Cummings SR, Harris T, Simonsick E, Satterfield S, Ayonayon H, Yaffe K. Chronic kidney disease and cognitive impairment in the elderly: the health, aging, and body composition study. J Am Soc Nephrol. 2005;16(7):2127-33.

13. Deva R, Alias MA, Colville D, Tow FK, Ooi QL, Chew S, Mohamad N, Hutchinson A, Koukouras I, Power DA, et al. Vision-threatening retinal abnormalities in chronic kidney disease stages 3 to 5. Clin J Am Soc Nephrol. 2011;6(8):1866-71.

14. Duenhas MR, Draibe SA, Avesani CM, Sesso R, Cuppari L. Influence of renal function on spontaneous dietary intake and on nutritional status of chronic renal insufficiency patients. Eur J Clin Nutr. 2003;57(11):1473-8.

15. Morley JE, Abbatecola AM, Argiles JM, Baracos V, Bauer J, Bhasin S, Cederholm T, Coats AJ, Cummings SR, Evans WJ, et al. Sarcopenia with limited mobility: an international consensus. J Am Med Dir Assoc. 2011; 12(6):403-9.

16. Foley RN, Wang C, Ishani A, Collins AJ, Murray AM. Kidney function and sarcopenia in the United States general population: NHANES III. Am J Nephrol. 2007;27(3):279-86.

17. Stevens PE, Lamb EJ, Levin A. Integrating guidelines, CKD, multimorbidity, and older adults. Am J Kidney Dis. 2015;65(3):494-501. https://doi.org/10. 1053/j.jajkd.2014.09.024. Epub 2014 Dec 4.

18. Levey AS, Inker LA, Coresh J. GFR estimation: from physiology to public health. Am J Kidney Dis. 2014;63(5):820-34.

19. Corsonello A, Tap L, Roller-Wirnsberger R, Wirnsberger G, Zoccali C, Kostka T, Guligowska A, Mattace-Raso F, Gil P, Fuentes LG, et al. Design and methodology of the screening for CKD among older patients across Europe (SCOPE) study: a multicenter cohort observational study. BMC Nephrol. 2018;19(1):260.

\section{Publisher's Note}

Springer Nature remains neutral with regard to jurisdictional claims in published maps and institutional affiliations.

Ready to submit your research? Choose BMC and benefit from:
- fast, convenient online submission
- thorough peer review by experienced researchers in your field
- rapid publication on acceptance
- support for research data, including large and complex data types
- gold Open Access which fosters wider collaboration and increased citations
- maximum visibility for your research: over 100M website views per year
At BMC, research is always in progress.
Learn more biomedcentral.com/submissions

